Pacific Journal of Mathematics

A SHORT PROOF OF ISBELL'S ZIGZAG THEOREM 


\title{
A SHORT PROOF OF ISBELL'S ZIGZAG THEOREM
}

\section{Peter M. Higgins}

\begin{abstract}
Isbell's Zigzag Theorem, which characterizes semigroup dominions (defined below) by means of equations, has several proofs. We give a short proof of the theorem from first principles.
\end{abstract}

The original proof Isbell [4] and that of Philip [6] are topological in flavour. The algebraic proofs of Howie [2] and Storrer [8] are based on work by Stenstrom [7] on tensor products of monoids. Yet another proof, using the geometric approach of regular diagrams, is due to David Jackson [5]. This latter approach also employs HNN extensions of semigroups to solve the problem. In this note we follow Jackson's lead in using what is essentially a HNN extension for our embedding (instead of the more intractable free product with amalgamation) to derive a short and direct proof of the Zigzag Theorem.

Following Howie and Isbell [3] we say that a subsemigroup $U$ of a semigroup $S$ dominates an element $d \in S$ if for every semigroup $T$ and all morphisms $\phi_{1}: S \rightarrow T, \phi_{2}: S \rightarrow T, \phi_{1}\left|U=\phi_{2}\right| U$ implies that $d \phi_{1}=d \phi_{2}$. The set of all elements in $S$ dominated by $U$ is called the dominion of $U$ in $S$; it is obviously a subsemigroup of $S$ containing $U$, and we denote it by $\operatorname{Dom}(U, S)$. Dominions are connected with epimorphisms (pre-cancellable morphisms) by the fact that a morphism $\alpha: S \rightarrow T$ is epi iff $\operatorname{Dom}(S \alpha, T)=T$.

IsBell's ZigzAG THEOREM. Let $U$ be a subsemigroup of $S$. Then $d \in \operatorname{Dom}(U, S)$ if and only if $d \in U$ or there exists a sequence of factorizations of $d$ as follows:

$$
d=u_{0} y_{1}=x_{1} u_{1} y_{1}=x_{1} u_{2} y_{2}=x_{2} u_{3} y_{2}=\cdots=x_{m} u_{2 m-1} y_{m}=x_{m} u_{2 m},
$$

where

$$
\begin{gathered}
u_{i} \in U, \quad x_{i}, y_{i} \in S, \quad u_{0}=x_{1} u_{1}, \quad u_{2 i-1} y_{i}=u_{2 i} y_{i+1}, \\
x_{i} u_{2 i}=x_{i+1} u_{2 i+1} \quad(1 \leq i \leq m-1) \quad \text { and } u_{2 m-1} y_{m}=u_{2 m} .
\end{gathered}
$$

Such equations are known as a zigzag in $S$ over $U$ with value $d$, length $m$, and spine the list $u_{0}, u_{1}, \ldots, u_{2 m}$. For a survey on 
epimorphisms and semigroup amalgams featuring applications of the Zigzag Theorem see Higgins [1].

We give a new proof of the forward implication in the theorem; the reverse implication follows by a straightforward manipulation of the zigzag.

Suppose that $d \in \operatorname{Dom}(U, S) \backslash U$. Form a semigroup $H$ by adjoining a new element $t$ to $S$ subject to the relations $t^{2}=1, t u=u t$, $t u t=u$ for all $u \in U$. Define the morphisms $\phi_{1}, \phi_{2}: S \rightarrow H$ by $s \phi_{1}=s$ and $s \phi_{2}=t s t$ (indeed $\phi_{1}$ and $\phi_{2}$ are embeddings). Clearly $\phi_{1}\left|U=\phi_{2}\right| U$ so that $t d t=d$, or what is the same, $t d=d t$ in $H$. We prove that this latter equation implies that $d$ is the value of some zigzag in $S$ over $U$.

Since $t d=d t$ there is a sequence of transitions of minimal length $I: t d \rightarrow \cdots \rightarrow d t$ where each transition $p w q \rightarrow p w^{\prime} q\left(p, w, w^{\prime}, q \in\right.$ $H)$ is either a $t$-transition, i.e., involves a relation in which $t$ occurs, or is a refactorization, i.e., $w=w^{\prime}$ in $S$. We claim that no transition in $I$ involves any of the relations $t^{2}=1$ or $t u t=u(u \in U)$. Suppose to the contrary that $I$ has a transition $\alpha: p q \rightarrow p t^{2} q(p, q \in$ $H)$. Clearly $\alpha$ is not the final transition of $I$, so consider the next transition $\beta: p t^{2} q \rightarrow$. Suppose that the right-hand side of $\beta$ has one of the forms
(i) $p q$;
(ii) $p^{\prime} t^{2} q$;
(iii) $p t^{2} q^{\prime}$.

In the first case the two transitions cancel, while in cases (ii) and (iii) $\alpha$ and $\beta$ can be performed in the opposite order without changing the net effect. If $\beta$ does not have one of these forms then either (iv) the product $p$ has the form $p=p^{\prime} u$ or $p^{\prime} t u(u \in U)$ and the right side has the form $p^{\prime} t u t q$ or $p^{\prime} u t q$ or $(\mathrm{v})$ a similar remark applies to $q$. In this case the pair of transitions $\alpha, \beta$ could be replaced by the single transition $p^{\prime} u q \rightarrow p^{\prime} t u t q$ or $p^{\prime} t u q \rightarrow p^{\prime} u t q$ (with a similar remark applying to case (v)). Therefore cases (i), (iv) and (v) contradict our minimum length assumption, whence it follows that all transitions of $I$ of the form $p q \rightarrow p t^{2} q$ can be taken to appear at the end of $I$, and thus there are none.

Next suppose that $\alpha$ has the form $p u q \rightarrow p t u t q$, and once again consider the following transition $\beta$. If $p$ has the form $p^{\prime} v$ or $p^{\prime} t v$ $(v \in U)$ then $\beta$ could have the form $p^{\prime} v t u t q \rightarrow p^{\prime}$ tvutq or $p^{\prime} t v t u t q \rightarrow$ $p^{\prime} v u t q$; but in that case the pair $\alpha, \beta$ could be replaced by the single transition $p^{\prime} v u q \rightarrow p^{\prime} t v u t q$ or $p^{\prime} t v u q \rightarrow p^{\prime} v u t q$. A similar remark applies if $q$ has the form $v q^{\prime}$ or $v t q^{\prime}$. If $p$ has the form $p^{\prime} t$ then $\beta$ could have the form $p^{\prime} t t u t q \rightarrow p^{\prime} u t q$; but again it would then 
be possible to shorten $I$ by replacing our pair $\alpha, \beta$ with the single transition $p^{\prime} t u q \rightarrow p^{\prime} u t q$; and again a similar remark applies to $q$. Another possibility for $\beta$ is $p t u t q \rightarrow p u t^{2} q$ or $p t u t q \rightarrow p t^{2} u q$, but here again $\alpha$ and $\beta$ could be replaced by just one transition. The remaining possibilities for $\beta$ ( $\beta$ cancels $\alpha$, or $\beta$ involves only the product $p$ or only the product $q$ ) are disposed of as in the previous paragraph, thus establishing the claim.

Call a $t$-transition of the form $p u t q \rightarrow p t u q \quad[p t u q \rightarrow p u t q]$ a left [right] transition, so that our sequence $I$ consists entirely of refactorizations and left and right transitions with exactly one occurrence of the symbol $t$ in each word of $I$. Suppose that $p t q$ is a product occurring in $I$, and that the next $t$-transition in the sequence is a left transition. We claim that we may assume that this left transition occurs immediately, or is preceded by just one refactorization of the form $p t q \rightarrow p^{\prime} u t q$, for it is clear that any refactorization of $p$ can be performed in one step, while any refactorization of $q$ can be delayed until after the left transition. Next suppose that $I$ contains two left transitions with no intervening right transition, which we may assume have the form $p u t q \rightarrow p t u q \rightarrow p^{\prime} v t u q \rightarrow p^{\prime} t v u q$ $(u, v \in U)$, or simply the form $p^{\prime} v u t q \rightarrow p^{\prime} v t u q \rightarrow p^{\prime} t v u q$. In the latter case the pair of transitions can be replaced by a single left transition, while the three transitions of the first case can be replaced by two: $p u t q \rightarrow p^{\prime} v u t q \rightarrow p^{\prime} t v u q$. Coupling all this with similar arguments for right transitions allows us to conclude that $I$ consists of alternate left and right transitions, separated by single refactorizations; furthermore the first $t$-transition is right and the final $t$-transition is right. The sequence $I$ therefore implies equalities in $H$ of the form:

$$
\begin{aligned}
t d & =t u_{0} y_{1}=u_{0} t y_{1}=x_{1} u_{1} t y_{1}=x_{1} t u_{1} y_{1}=x_{1} t u_{2} y_{2}=x_{1} u_{2} t y_{2} \\
& =x_{2} u_{3} t u_{2}=\cdots=x_{m-1} u_{2 m-2} t y_{m}=x_{m} u_{2 m-1} t y_{m} \\
& =x_{m} t u_{2 m-1} y_{m}=x_{m} t u_{2 m}=x_{m} u_{2 m} t=d t,
\end{aligned}
$$

for some $m \geq 1, u_{i} \in U(1 \leq i \leq 2 m) \quad x_{i}, y_{i} \in S^{1}$, and $u_{0}=x_{1} u_{1}$,

$$
\begin{gathered}
u_{2 i-1} y_{i}=u_{2 i} y_{i+1} \quad x_{i} u_{2 i}=x_{i+1} u_{2 i+1}, \quad(1 \leq i \leq m-1) \quad \text { and } \\
u_{2 m-1} y_{m}=u_{2 m} .
\end{gathered}
$$

In fact $x_{i}, y_{i} \in S$ for if $x_{i}=1$ then in $S$ we have

$$
d=u_{0} y_{1}=x_{1} u_{1} y_{1}=x_{1} u_{2} y_{2}=\cdots=u_{2 i} y_{i+1}=\cdots=x_{m} u_{2 m} ;
$$

and so I could be shortened by beginning with $t d \rightarrow t u_{2 i} y_{i+1}$, with a similar remark applying if some $y_{i}=1$. Hence $d$ is the value of a zigzag in $S$ over $U$, thus completing the proof. 


\section{REFERENCES}

[1] P. M. Higgins, Epimorphisms and amalgams, Colloquium Mathematicum, LVI (1988), 1-17.

[2] J. M. Howie, An Introduction to Semigroup Theory, London Math. Soc. Monographs 7, Academic Press, 1976.

[3] J. M. Howie and J. R. Isbell, Epimorphisms and dominions II, J. Algebra, 6 (1967), 7-21.

[4] J. R. Isbell, Epimorphisms and dominions, Proc. of the Conference on Categorical Algebra, La Jolla, (1965), Lange and Springer, Berlin 1966, 232-246.

[5] D. Jackson, Regular diagrams for the study of algebraic semigroups, manuscript.

[6] J. M. Philip, A proof of Isbell's Zigzag Theorem, J. Algebra, 32 (1974), 328-331.

[7] B. Stenstrom, Flatness and localization over monoids, Math. Nachr., 48 (1971), 315-334.

[8] H. H. Storrer, An algebraic proof of Isbell's Zigzag Theorem, Semigroup Forum, 12 (1976), 83-88.

Received July 17, 1989.

Royal Melbourne Institute of Technology

GPOB 2476V

Melbourne, Vic. 3001, Australia 


\section{PACIFIC JOURNAL OF MATHEMATICS EDITORS}

\author{
V. S. VARADARAJAN \\ (Managing Editor) \\ University of California \\ Los Angeles, CA 90024-1555-05 \\ Herbert Clemens \\ University of Utah \\ Salt Lake City, UT 84112 \\ Thomas ENRIGHT \\ University of California, San Diego \\ La Jolla, CA 92093
}

R. FINN

Stanford University

Stanford, CA 94305

Hermann FlaschKa

University of Arizona

Tucson, AZ 85721

VAUGHAN F. R. Jones

University of California

Berkeley, CA 94720

STEVEN KERCKHOFF

Stanford University

Stanford, CA 94305
C. C. MOORE

University of California

Berkeley, CA 94720

Martin SCharlemanN

University of California

Santa Barbara, CA 93106

HAROLd STARK

University of California, San Diego

La Jolla, CA 92093

\section{ASSOCIATE EDITORS}
R. ARENS
E. F. BECKENBACH
B. H. NeumanN
F. WoLF
(1904-1989)
K. YoshidA (1906-1982)

\section{SUP}

UNIVERSITY OF ARIZONA

UNIVERSITY OF BRITISH COLUMBIA

CALIFORNIA INSTITUTE OF TECHNOLOGY

UNIVERSITY OF CALIFORNIA

MONTANA STATE UNIVERSITY

UNIVERSITY OF NEVADA, RENO

NEW MEXICO STATE UNIVERSITY

OREGON STATE UNIVERSITY
UNIVERSITY OF OREGON

UNIVERSITY OF SOUTHERN CALIFORNIA

STANFORD UNIVERSITY

UNIVERSITY OF HAWAII

UNIVERSITY OF TOKYO

UNIVERSITY OF UTAH

WASHINGTON STATE UNIVERSITY

UNIVERSITY OF WASHINGTON 


\section{Pacific Journal of Mathematics}

Vol. 144, No. $1 \quad$ May, 1990

Wojciech Chojnacki, On some totally ergodic functions $\ldots \ldots \ldots \ldots \ldots \ldots 1$

Steven R. Costenoble, Stefan Waner and G. S. Wells, Approximating

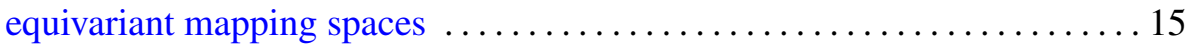

Peter Michael Higgins, A short proof of Isbell's zigzag theorem . . . . . . . 47

Harold H. Johnson, The absolute invariance of conservation laws ........5 51

Edgar Kann, Infinitesimal rigidity of almost-convex oriented polyhedra of arbitrary Euler characteristic $\ldots \ldots \ldots \ldots \ldots \ldots \ldots \ldots \ldots \ldots \ldots \ldots \ldots$

Alan Van Lair, Uniqueness for a nonlinear abstract Cauchy problem . . . . . 105

John B. Little and Kathryn A. Furio, On the distribution of Weierstrass points on irreducible rational nodal curves $\ldots \ldots \ldots \ldots \ldots \ldots \ldots \ldots \ldots 131$

J. S. Okon and Louis Jackson Ratliff, Jr., Reductions of filtrations . . . . . 137

Janusz Pawlikowski, Small subset of the plane which almost contains almost all Borel functions ............................... 155

Sergio A. Tozoni, Vector singular integral operators on a local field ....... 161 John Bason Wagoner, Triangle identities and symmetries of a subshift of

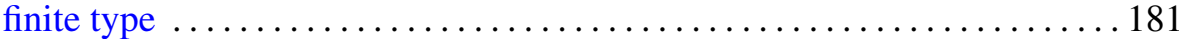

